\title{
Etude quantitative de l'adsorption et de la désorption de l'UKJ-1506 dans le sol
}

\author{
Paul JAMET
}

I.N.R.A., Service de Chimie des Pesticides, Station de Phytopharmacie et d'Ecotoxicologie, F 78000 Versailles

\begin{abstract}
Une méthode du type agitation a été utilisée pour étudier les caractéristiques de l'adsorption et de la désorption de l'UKJ-1506 (thiopyrimidine herbicide). L'étude de la cinétique d'adsorption a montré qu'après 4 heures d'agitation l'équilibre d'adsorption est atteint. Les paramètres $\mathrm{K}$ et $1 / \mathrm{n}$ des isothermes d'adsorption et de désorption répondant au modèle empirique de FREUNDLICH $\mathrm{x} / \mathrm{m}=\mathrm{KC}^{1 / \mathrm{n}}$ ont été déterminés par régression non linéaire. Le coefficient d'adsorption $\mathrm{K}$ est d'autant plus grand que la teneur du sol en matières organiques est plus élevée. Les isothermes d'adsorption ne sont pas linéaires $(1 / n<1)$. Pour tous les sols utilisés, les paramètres $\mathrm{K}$ et $1 / \mathrm{n}$ sont indépendants de la gamme de concentrations utilisée pour leur détermination ; l'adsorption de l'UKJ-1506 est un phénomène réversible. L'intérêt de la régression non linéaire pour les déterminations des paramètres $K$ et $1 / n$ est souligné.
\end{abstract}

Mots clés additionnels : Herbicide, cinétique d'adsorption, isotherme d'adsorption, isotherme de désorption, régression non-linéaire.

The characteristics of the adsorption-desorption of UKJ-1506 (thiopyrimidine herbicide) were studied using a batch technique. Study of the adsorption kinetics showed that equilibrium adsorption was reached after 4-h shaking. Non-linear regression was used to estimate the parameters $K$ and $1 / n$ of the FreundLicH equation $\left(\mathrm{x} / \mathrm{m}=\mathrm{KC}^{1 / \mathrm{n}}\right)$. The adsorption coefficient $\mathrm{K}$ increased with higher soil organic matter content. The adsorption isotherms were not linear $(1 / n<1)$. For all the soils tested parameters $K$ and $1 / n$ were independent of the concentration range used. UKJ-1506 adsorption could be reversed. The value of non-linear regression for estimating $\mathrm{K}$ and $1 / \mathrm{n}$ is stressed.

Additional key words : Herbicide, adsorption kinetics, adsorption isotherm, desorption isotherm, nonlinear regression.

\section{INTRODUCTION}

L'étude quantitative de l'adsorption et de la désorption d'une matière active est une étape importante pour la compréhension de son comportement dans le sol. Si les techniques dérivées de la chromatographie (couches minces et colonnes de sol) sont très utilisées pour étudier la mobilité d'un produit phytosanitaire dans le sol, seule la détermination de la quantité adsorbée permet une estimation fiable de la fraction biodisponible ; en effet, c'est cette fraction qui, présente dans la solution du sol, assure l'efficacité du traitement. La fraction adsorbée par les constituants du sol est provisoirement soustraite à l'action des phénomènes de transport et de dégradation ; elle sera le plus souvent libérée progressivement dans la solution du sol prolongeant ainsi la rémanence du produit. (CALVET \& JAMET, 1979 ; JAMET \& WOLFF, 1981).
L'étude quantitative de l'adsorption et de la désorption est classiquement réalisée au laboratoire à l'aide d'une technique du type agitation ; le principe de cette technique, facile à mettre en œuvre, repose sur celui de la détermination d'un coefficient de partage : une quantité de sol est agitée en présence d'une solution aqueuse de la molécule étudiée. La diminution de la concentration de la solution permet de calculer la quantité de produit adsorbé par l'échantillon de sol. En répétant cette opération pour différentes concentrations initiales de la phase aqueuse on peut alors déterminer l'allure de l'isotherme d'adsorption; le modèle empirique de FreundLICH (équation 2) est généralement utilisé pour décrire les isothermes ainsi obtenues.

Si la technique utilisée présente certains inconvénients analysés par ailleurs (GREEN \& YAMANE, 1971 ; CALVET et al., 1980b), elle permet cependant d'obte- 
nir une estimation satisfaisante des paramètres numériques du modèle utilisé. Ces paramètres servent à quantifier l'adsorption (tel $\mathrm{K}$ le coefficient d'adsorption) ou à qualifier l'allure de l'isotherme donc les interactions adsorbant-adsorbé (tel $1 / \mathrm{n}$ du modèle de FREUNDLICH). Ces paramètres peuvent aussi être introduits dans des modèles mathématiques de prévision du transfert ou de la dégradation dans le sol. Le plus souvent ces paramètres numériques sont estimés par régression linéaire après linéarisation du modèle par une transformation mathématique appropriée.

Dans le cadre d'une étude classique de l'adsorption et de la désorption consacrée à l'UKJ-1506 nous avons voulu souligner l'intérêt de l'emploi :

- d'une large gamme de concentrations initiales pour la détermination des isothermes d'adsorption et de désorption,

- de la régression non linéaire pour l'estimation des paramètres numériques des modèles utilisés (cinétique et isotherme d'adsorption).

L'UKJ-1506, actuellement connu sous le nom de tioclorine, est un herbicide de la famille des thiopyrimidines développé au début des années 80 (BOUTEMY, 1980). Cette étude quantitative de l'adsorption et de la désorption de cet herbicide vient en complément de l'étude de son entraînement par lessivage réalisée précédemment (JAMET \& WOLFF, 1981). Trois aspects sont abordés ici :

- étude de la cinétique d'adsorption : cette rapide étude n'a pour but que l'estimation du temps nécessaire pour parvenir à l'équilibre, estimation indispensable pour la suite,

- détermination des isothermes d'adsorption: il s'agit de caractériser la situation d'équilibre par l'allure des isothermes et d'estimer le coefficient d'adsorption $\mathrm{K}$ pour comparer l'adsorption de la tioclorine par différents sols.

- détermination des isothermes de désorption : la réversibilité de l'adsorption est appréciée à l'aide d'une seule désorption.

\section{MATÉRIEL ET MÉTHODES}

\section{A. La matière active utilisée}

L'UKJ-1506 utilisé est en réalité un mélange de deux isomères, dans les proportions suivantes :<smiles>CSc1c(N)nc(N)nc1Cl</smiles>

$\mathbf{A}$

$\mathbf{B}$<smiles>CSc1c(N)nc(Cl)nc1N</smiles>

A : chloro-6 diamino-2,4 méthylthio-5 pyrimidine (90 p. 100).

B : chloro-2 diamino-4,6 méthylthio-5 pyrimidine (10 p. 100).

L'activité herbicide de l'UKJ-1506 est due à l'isomère chloro-6 (A) qui représente 90 p. 100 du mélange mis à notre disposition pour cette étude.
La molécule de ${ }^{14} \mathrm{C}-2 \mathrm{UKJ}-1506$ fournie par la société obtentrice a été synthétisée dans les laboratoires du CEA à Saclay; son activité spécifique est de $888 \mathrm{MBq} / \mathrm{mmole}(\mathrm{PM}=190,5)$. La solubilité dans l'eau de l'UKJ-1506 est de $500 \mathrm{mg} / \mathrm{l}$ à $20^{\circ} \mathrm{C}$.

\section{B. Le choix des sols}

Sept des huit sols utilisés dans cette étude proviennent des parcelles expérimentales situées dans différentes régions où furent réalisés des essais d'efficacité de l'UKJ-1506 ; le huitième sol provient de la parcelle des Closeaux de l'INRA-Versailles. Les principales caractéristiques physico-chimiques de ces sols, déterminées par le laboratoire des sols de l'I.N.R.A. à Arras, sont regroupées dans le tableau 1. Mis à part le sol 7 (Milly-la-Forêt) qui est un limon, tous les autres sols sont des limons fins qui diffèrent par leur $\mathrm{pH}$ (de 5,8 à 7,8 ) et surtout par leur teneur en matières organiques (de 1,32 à 5,37 p. 100). Deux d'entre eux, les sols 3 et 4 (Azy et Cuinchy) contiennent respectivement 4,02 et 5,37 p. 100 de matières organiques alors que la teneur des autres sols est inférieure à 2 p. 100 .

\section{Etude de la cinétique d'adsorption}

Deux cents grammes de sol sont agités en permanence durant $24 \mathrm{~h}$ en présence de $500 \mathrm{ml}$ d'une solution aqueuse de ${ }^{14} \mathrm{C}-2$ UKJ-1506. Plusieurs séries de deux prélèvements (d'environ $10 \mathrm{ml}$ chacun) sont effectuées à $5 ; 15 ; 30 ; 45 \mathrm{mn} ; 1 ; 2 ; 4 ; 8$ et $24 \mathrm{~h}$. Ceux-ci sont immédiatement centrifugés durant $5 \mathrm{mn}$ à $10000 \mathrm{~g}$; trois pipettages de $1 \mathrm{ml}$ sont alors effectués pour déterminer la concentration d'herbicide de la phase aqueuse. Cette étude de la cinétique d'adsorption est réalisée avec deux sols ( 1 et 4$)$, à deux concentrations initiales ( 1 et $50 \mu \mathrm{g} / \mathrm{ml}$ ) ; dans chaque cas deux répétitions sont effectuées. Le choix de ces deux sols (1 et 4) se justifie par leur provenance (région céréalière pour le sol 1) ou par leur teneur en matières organiques: le sol 4 est, des sols étudiés, celui qui a la plus forte teneur en matières organiques; le sol 1 ayant une des plus faibles teneurs (tabl. 1).

\section{Détermination des isothermes d'adsorption}

Dans des pots en polycarbonate de $250 \mathrm{ml}$, on met en présence une quantité $\mathrm{m}$ de $\mathrm{sol}(\mathrm{m}=20 \mathrm{~g})$ et un volume $\mathrm{V}(\mathrm{V}=50 \mathrm{ml})$ d'une solution aqueuse de ${ }^{14} \mathrm{C}$ $2 \mathrm{UKJ}-1506$ à la concentration $\mathrm{Co}(\mu \mathrm{g} / \mathrm{ml})$. Ces pots, maintenus à température ambiante $\left(20^{\circ} \mathrm{C} \pm 2{ }^{\circ} \mathrm{C}\right)$, sont agités mécaniquement pendant $4 \mathrm{~h}$; ils sont ensuite soumis à une centrifugation d'environ $10000 \mathrm{~g}$ durant $10 \mathrm{mn}$ dans une centrifugeuse à température stabilisée $\left(20^{\circ} \mathrm{C} \pm 1{ }^{\circ} \mathrm{C}\right)$. La concentration C d'UKJ-1506 restant dans la phase aqueuse ainsi séparée est déterminée sur 3 fois $1 \mathrm{ml}$. Deux répétitions sont effectuées pour chacune des 14 concentrations Co suivantes : 0,$1 ; 0,2 ; 0,4 ; 0,8 ; 1 ; 2 ; 4 ; 8$; $10 ; 20 ; 40 ; 80 ; 160$ et $320 \mu \mathrm{g} / \mathrm{ml}$; ainsi chaque isotherme est calculée à partir de 28 points expérimentaux. 
TABLEAU 1

Principales caractéristiques des sols.

Main characteristics of the soils used.

\begin{tabular}{|c|c|c|c|c|c|c|c|c|}
\hline \multirow{2}{*}{ Caractéristiques } & \multicolumn{8}{|c|}{ SOLS } \\
\hline & 1 & 2 & 3 & 4 & 5 & 6 & 7 & 8 \\
\hline \multicolumn{9}{|l|}{ Granulométrie $(\%$ oo $)$} \\
\hline$<2 \mu \mathrm{m}$ & 171 & 112 & 226 & 182 & 182 & 129 & 191 & 205 \\
\hline $2-20 \mu \mathrm{m}$ & 276 & 347 & 452 & 302 & 257 & 297 & 169 & 211 \\
\hline $20-50 \mu \mathrm{m}$ & 263 & 394 & 167 & 359 & 476 & 391 & 226 & 427 \\
\hline $50-200 \mu \mathrm{m}$ & 196 & 112 & 34 & 117 & 73 & 144 & 327 & 125 \\
\hline $200-2000 \mu \mathrm{m}$ & 94 & 35 & 121 & 40 & 12 & 39 & 87 & 27 \\
\hline Matières organiques $\%$ & 1,54 & 1,79 & 4,02 & 5,37 & 1,48 & 1,57 & 1,32 & 1,94 \\
\hline $\begin{array}{l}\text { Capacité d'échange } \\
\mathrm{m} \text {. équiv. }(100 \mathrm{~g})\end{array}$ & 11,7 & 7,4 & 22,7 & 19,0 & 12,1 & 7,2 & 11,3 & 10,0 \\
\hline $\mathrm{pH}(\mathrm{eau})$ & 7,6 & 6,4 & 7,8 & 7,7 & 7,1 & 5,8 & 7,0 & 6,4 \\
\hline Classification & limon fin & limon fin & limon fin & limon fin & limon fin & limon fin & limon & limon fin \\
\hline
\end{tabular}

1 : Laval-en-Brie (77)

2: Villars-Les-Dombes (01)

3 : Azy (18)

4 : Cuinchy (62)
$5:$ Lizy-sur-Ourcq (77)

6 : Meximieux (01)

7 : Milly-la-Forêt $(91)$

$8:$ INRA-Versailles (78)

\section{E. Détermination des isothermes de désorption}

Après prélèvement des $3 \mathrm{ml}$ de solution pour déterminer la quantité d'UKJ-1506 adsorbé, les pots sont vidés de leur solution aqueuse ; celle-ci est alors remplacée par $50 \mathrm{ml}$ d'eau distillée. On procède ensuite comme pour l'étude de l'adsorption. Pour les raisons énoncées dans le paragraphe suivant, et en l'absence de données sur la cinétique de désorption de l'UKJ1506, nous avons retenu un temps d'agitation cinq fois plus élevé $(20 \mathrm{~h})$ pour l'étude de la désorption que' pour l'étude de l'adsorption. De plus, lors de la mesure de l'adsorption, les sols retiennent un volume $\mathrm{v}(\mathrm{ml})$ de solution aqueuse à la concentration $\mathrm{C}$. $\mathrm{C} \mathrm{C}$ volume, qui ne peut pas être éliminé avant d'ajouter les $50 \mathrm{ml}$ d'eau distillée, est mesuré par pesée ; variant selon les sols, ce volume est pris en compte lors du calcul de $\mathrm{C}^{\prime}$, la concentration de la phase aqueuse après désorption (JAMET \& PIEDALlU, 1975b).

\section{F. Mesure de la radioactivité dans les phases aqueuses}

Dans le sol, la demi-vie de l'UKJ-1506 estimée par KERHOAS \& DUBROCA, 1984, est d'environ deux semaines. Comme la durée totale de nos expérimentations n'excède pas $48 \mathrm{~h}$, la dégradation de l'UKJ-1506 peut être considérée comme très limitée. C'est pourquoi les concentrations $\mathrm{Co}, \mathrm{C}$ et $\mathrm{C}^{\prime}$ précédemment définies sont déterminées en faisant la moyenne des mesures de radioactivité sur 3 fois $1 \mathrm{ml}$ des solutions aqueuses correspondantes auxquels on ajoute $4 \mathrm{ml} \mathrm{du}$ liquide scintillant suivant :

$\begin{array}{lll}\text { diméthyl POPOP } & 300 \mathrm{mg} \\ \text { PPO } & : & 7 \mathrm{mg} \\ \text { naphtalène } & : 100 \mathrm{~g} \\ \text { 1,4-dioxanne } & : 1000 \mathrm{ml}\end{array}$

Les mesures de radioactivité sont effectuées à l'aide d'un spectrophotomètre à scintillateur liquide INTERTECHNIQUE IN 4221.

\section{G. Modélisation des phénomènes étudiés}

Pour modéliser la cinétique d'adsorption de l'UKJ1506 nous avons utilisé un modèle hyperbolique dérivé de celui proposé par BIGGAR et al. (1978) :

$$
\mathrm{Q}(\mathrm{t})=(\mathrm{Qmax} \cdot \mathrm{t}) /(\mathrm{k}+\mathrm{t})
$$

où :

$\mathrm{Q}(\mathrm{t})$ représente la quantité adsorbée à l'instant $\mathrm{t}$, Qmax. la quantité maximale adsorbée,

$\mathrm{k}$ est une constante; plus la valeur de $\mathrm{k}$ est faible, plus le produit s'adsorbe rapidement.

Ce modèle, purement empirique, permet une estimation fiable de la quantité maximale adsorbée $(t=\infty)$; on peut donc en déduire le temps au bout duquel l'équilibre d'adsorption peut être considéré comme atteint.

La modélisation des isothermes d'adsorption et de désorption repose sur l'utilisation du modèle empirique de FREUNDLICH :

$\mathrm{x} / \mathrm{m}=\mathrm{KC}^{1 / \mathrm{n}}$

dans lequel :

$\mathrm{x} / \mathrm{m}$ représente la quantité adsorbée à l'équilibre $(\mu \mathrm{g} / \mathrm{g})$,

C désigne la concentration d'herbicide à l'équilibre dans la solution $\mathrm{du}$ sol $(\mu \mathrm{g} / \mathrm{ml})$,

$\mathrm{K}$ est le coefficient d'adsorption ; plus sa valeur est élevée, plus l'adsorption est importante,

$1 / \mathrm{n}$ est un exposant dont la valeur, généralement inférieure à 1 , détermine l'allure de l'isotherme.

L'estimation des paramètres de ces deux modèles non linéaires est réalisée par une méthode d'optimisation numérique ; cette technique a nécessité l'utilisa- 
tion du programme HAUSS59 décrit par JOLIVET (1983). Ce programme, que nous avons implanté sur un microordinateur KONTRON PSI-80, est écrit en BASIC et utilise l'algorithme de GAUSS-MARQUARD.

\section{RÉSULTATS ET DISCUSSION}

\section{A. Cinétique d'adsorption}

Les résultats des modélisations sont regroupés dans le tableau 2 ; cependant pour en faciliter l'interprétation, 2 des 8 expériences réalisées sont présentées sous forme graphique (fig. 1, 2).

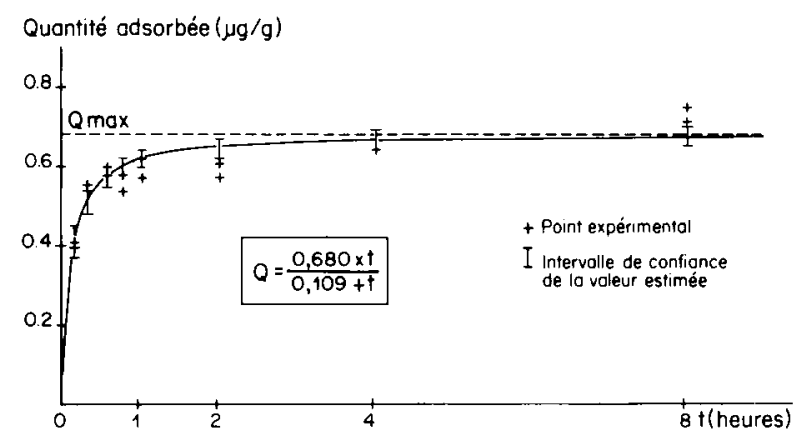

Figure 1

Modélisation de la cinétique d'adsorption de l'UKJ-1506 selon le modele hyperholique sur le sol I de Laval-en-Brie (77); concentration initiale : $1,02 \mu \mathrm{g} / \mathrm{ml}$.

Modeling of the adsorption kinetics of UKJ-1506 according 10 the hyperbolic model for soil 1 from Laval-en-Brie (77); initial concentration : $1.02 \mu \mathrm{g} / \mathrm{ml}$.

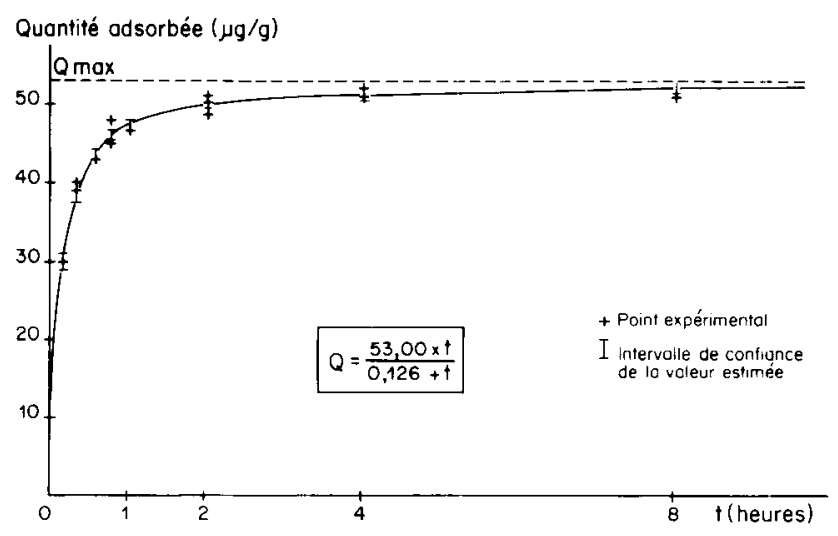

Figure 2

Modélisation de la cinétique d'adsorption de l'UKJ-1506 selon te modèle hyperbolique sur le sol 4 de Cuinchy (62); concentration initiale : $50,6 \mu \mathrm{g} / \mathrm{ml}$.

Modeling of the adsorption kinetics of UKJ-1506 according to the hyperbolic model for soil 4 from Cuinchy (62); initial concentration : $50.6 \mu \mathrm{g} / \mathrm{ml}$.

On observe une très rapide augmentation de la quantité adsorbée au cours des premières minutes de contact ; en effet, l'équilibre d'adsorption est pratiquement atteint au bout d'une heure sur le sol de Laval-en-Brie (fig. 1), et de 3 à $4 \mathrm{~h}$ sur le sol de Cuinchy (fig. 2). Au-delà, cette phase d'adsorption "rapide " est suivie d'une phase d'adsorption « lente » durant laquelle la quantité adsorbée ne croît que très lentement. A la vue de ces résultats un temps de contact de $4 \mathrm{~h}$ a été retenu pour tous les sols lors de la détermination des isothermes d'adsorption. Les différences observées (fig. 1,2) sont confirmées par les résultats des modélisations. En effet, un critère simple pour estimer le temps nécessaire pour parvenir à l'équilibre d'adsorption consiste à considérer l'instant t à partir duquel la quantité de pesticide adsorbé $\mathrm{Q}(\mathrm{t})$ est supérieure ou égale à (1-x) fois la quantité maximale adsorbée Qmax. D'après JAMET et al. (1984) le temps $t_{(1-x)}$ est donné par la relation :

$\mathrm{t}_{(1-\mathrm{x})}=\mathrm{k}[(1-\mathrm{x}) / \mathrm{x}]$

En prenant $\mathrm{x}=0,05$ on peut calculer $t_{95}$ à l'aide des valeurs de $\mathrm{k}$ du tableau 2 . La valeur maximale estimée pour $\mathrm{k}$ est de 0,197 ; elle conduit à une valeur de $t_{95}$ égale à $3,74 \mathrm{~h}$, d'où le choix qui a été fait pour le temps de contact $(4 \mathrm{~h})$.

Cependant, les valeurs de $k$, en particulier pour le sol de Laval-en-Brie peuvent varier considérablement d'une répétition à l'autre (tabl. 2). La validité de l'estimation de $\mathrm{k}$ dépend essentiellement de la précision des mesures effectuées au cours de la phase d'adsorption rapide. Malgré les précautions prises, 3 sources d'erreur peuvent exister :

- l'homogénéité du mélange sol-solution aqueuse

\section{TABLEAU 2}

Cinétique d'adsorption de l'UKJ-1506: paramètres du modèle hyperbolique $Q(t)=Q \max . t /(k+t)$ estimés pour différentes conditions expérimentales; chàque valeur estimée est accompagnée de son intervalle de confiance approché à $95 p .100$.

Adsorption kinetics of UKJ-1506: parameters of the hyperbolic model $Q(t)=Q m a x . t /(k+t)$ estimated for different experimental conditions; each estimated value is given with its $95 \%$ confidence interval.

\begin{tabular}{|c|c|c|c|c|c|}
\hline SOL & $\begin{array}{r}\text { Co } \\
\text { (2 répétit }\end{array}$ & ons) & $\begin{array}{c}Q \max . \\
(\mu \mathrm{g} / \mathrm{g})\end{array}$ & $\mathrm{k}$ & $\mathrm{CD}\left({ }^{*}\right)$ \\
\hline \multirow{4}{*}{$\begin{array}{c}1 \\
\text { Laval- } \\
\text { en- } \\
\text { Brie } \\
(77)\end{array}$} & \multirow{2}{*}{$\begin{array}{c}1 \\
\mu \mathrm{g} / \mathrm{ml}\end{array}$} & 1 & $\begin{array}{c}0,680 \\
(0,650-0,709)\end{array}$ & $\begin{array}{c}0,109 \\
(0,074-0,144)\end{array}$ & 0,8207 \\
\hline & & 2 & $\begin{array}{c}0,704 \\
(0,680-0,729)\end{array}$ & $\begin{array}{c}0,044 \\
(0,024-0,064)\end{array}$ & 0,6068 \\
\hline & \multirow{2}{*}{$\begin{array}{c}50 \\
\mu \mathrm{g} / \mathrm{ml}\end{array}$} & 1 & $\begin{array}{c}23,892 \\
(23,183-24,601)\end{array}$ & $\begin{array}{c}0,066 \\
(0,047-0,086)\end{array}$ & 0,8144 \\
\hline & & 2 & $\begin{array}{c}22,800 \\
(21,770-23,830)\end{array}$ & $\begin{array}{c}0,016 \\
(0,005-0,038)\end{array}$ & 0,1441 \\
\hline \multirow{4}{*}{$\begin{array}{c}4 \\
\text { Cuinchy } \\
(62)\end{array}$} & \multirow{2}{*}{$\begin{array}{c}1 \\
\mu \mathrm{g} / \mathrm{ml}\end{array}$} & 1 & $\begin{array}{c}1,624 \\
(1,586-1,662)\end{array}$ & $\begin{array}{c}0,067 \\
(0,051-0,082)\end{array}$ & 0,8758 \\
\hline & & 2 & $\begin{array}{c}1,638 \\
(1,602-1,675)\end{array}$ & $\begin{array}{c}0,192 \\
(0,168-0,216)\end{array}$ & 0,9748 \\
\hline & \multirow{2}{*}{$\begin{array}{c}50 \\
\mu \mathrm{g} / \mathrm{ml}\end{array}$} & 1 & $\begin{array}{c}53,002 \\
(52,208-53,796)\end{array}$ & $\begin{array}{c}0,126 \\
(0,113-0,139)\end{array}$ & 0,9793 \\
\hline & & 2 & $\begin{array}{c}53,674 \\
(51,682-55,666)\end{array}$ & $\begin{array}{c}0,197 \\
(0,157-0,238)\end{array}$ & 0,9284 \\
\hline
\end{tabular}

$\left(^{*}\right)$ Coefficient de détermination ; 16 degrés de liberté. 1 et 2 sont des répétitions.

Determination coefficient; 16 degrees of freedom. 1 and 2 are duplicates. 
au cours des premières minutes d'agitation n'est certainement pas parfaite,

- la centrifugation rapide effectuée pour les premiers prélèvements ne permet pas toujours une séparation correcte des phases liquide et solide,

- l'estimation du temps de contact effectif entre les constituants du sol et la solution aqueuse est difficile ; et ceci l'est d'autant plus pour les premières mesures. En effet, la centrifugation accroît le temps de contact d'une durée difficilement estimable; nous avons cependant décidé d'augmenter la valeur du temps d'agitation réelle (temps écoulé jusqu'au prélèvement) d'une valeur arbitraire de $5 \mathrm{mn}$ (correspondant au temps de centrifugation). Pour les premiers prélèvements le temps de centrifugation est loin d'être négligeable par rapport au temps d'agitation réelle avant prélèvement.

Contrairement aux valeurs de $\mathbf{k}$, les valeurs de Qmax, quantité maximale adsorbée, ne diffèrent jamais de manière significative d'une répétition à l'autre (tabl. 2). Ces estimations de Qmax fournissent d'ores et déjà des renseignements intéressants, soit en les comparant, soit en calculant les pourcentages d'UKJ-1506 adsorbé. Pour les concentrations de 1 et $50 \mu \mathrm{g} / \mathrm{ml}$, le sol de Laval-en-Brie adsorbe respectivement 27 et 19 p. 100 de l'herbicide en solution ; les valeurs correspondantes pour le sol de Cuinchy sont 64 et 43 p. 100 . Pour ces deux sols, les isothermes d'adsorption ne seront pas linéaires $(1 / n<1)$; et le coefficient d'adsorption du sol de Cuinchy sera plus élevé que celui du sol de Laval-en-Brie.

Ainsi, le modèle hyperbolique utilisé permet dans la plupart des cas une description satisfaisante du phénomène étudié, les valeurs du coefficient de détermina- tion sont supérieures à 0,8 voire à 0,9 sauf pour 2 répétitions (plus la valeur de ce coefficient est proche de l'unité, plus l'ajustement est satisfaisant).

\section{B. Les isothermes d'adsorption}

Disposant de 28 points expérimentaux, obtenus avec une gamme de concentrations initiales variant de 0,1 à $320 \mu \mathrm{g} / \mathrm{ml}$, nous avons effectué 4 séries d'ajustement : gamme de 0,1 à $\quad 1 \mu \mathrm{g} / \mathrm{ml}$ (10 points expérimentaux) gamme de 1 à $10 \mu \mathrm{g} / \mathrm{ml}$ (10 points expérimentaux) gamme de 10 à $320 \mu \mathrm{g} / \mathrm{ml}$ (12 points expérimentaux) gamme de 0,1 à $320 \mu \mathrm{g} / \mathrm{ml}$ (28 points expérimentaux)

Les valeurs estimées des paramètres $1 / \mathrm{n}$ et $\mathrm{K}$, obtenues pour chaque gamme, sont regroupées dans le tableau 4 ; les isothermes calculées avec la totalité des points expérimentaux sont représentées en figure 5. Les figures 3 et 4 , et le tableau 3 permettent de comparer les estimations des paramètres $K$ et $1 / n$ des isothermes d'adsorption et de désorption.

Considérons tout d'abord les isothermes d'adsorption calculées avec la totalité des valeurs expérimentales. L'équation de FREUNDLICH permet une description très satisfaisante de la variation de la quantité adsorbée en fonction de la concentration à l'équilibre : les valeurs du coefficient de détermination sont toutes très proches de l'unité ( $>0,98$ voire 0,99 ) (tabl. 3 ). Pour tous les sols, les valeurs estimées de $1 / n$ sont inférieures à l'unité ; cela signifie que la quantité de pesticide adsorbée $(\mathrm{x} / \mathrm{m})$ croît proportionnellement moins vite que la concentration à l'équilibre $\mathrm{C}$. Parmi toutes les valeurs de $1 / n$, seule celle correspondant au sol de Versailles diffère significativement des autres qui peu-

TABLEAU 3

Comparaison des paramètres d'adsorption et de désorption de l'UKJ-1506 calculés d'après le modèle empirique de $F_{R E U N D L I C H ~}\left(x / m=K C C^{l / n}\right)$. La valeur estimée de chaque paramètre est accompagnée de son intervalle de confiance approché à 95 p. 100.

Comparison of the adsorption and desorption parameters of UKJ-1506 calculated with the empirical FREUNDLICH model $(x / \mathrm{m}=\mathrm{KC} / \mathrm{n})$. The estimated value for each parameter is given with its $95 \%$ confidence interval.

\begin{tabular}{|c|c|c|c|c|c|c|}
\hline \multirow[b]{2}{*}{ SOLS } & \multicolumn{3}{|c|}{ ADSORPTION } & \multicolumn{3}{|c|}{ DÉSORPTION } \\
\hline & $\mathrm{K}$ & $1 / \mathrm{n}$ & $\mathrm{CD}$ (a) & $\mathrm{K}^{\prime}$ & $1 / n^{\prime}$ & CD (a) \\
\hline 1 Laval-en-Brie (77) & $\begin{array}{c}1,246 \\
(0,782-1,710)\end{array}$ & $\begin{array}{c}0,834 \\
(0,766-0,903)\end{array}$ & 0,9859 & $\begin{array}{c}1,400 \\
(0,645-2,152)\end{array}$ & $\begin{array}{c}0,926 \\
(0,789-1,062)\end{array}$ & 0,9573 \\
\hline 2 Villars-les-Dombes $(01)$ & $\begin{array}{c}1,165 \\
(0,842-1,488) \\
\end{array}$ & $\begin{array}{c}0,873 \\
(0,822-0,924)\end{array}$ & 0,9929 & $\begin{array}{c}1,435 \\
(0,861-2,008)\end{array}$ & $\begin{array}{c}0,898 \\
(0,799-0,997)\end{array}$ & 0,9752 \\
\hline 3 Azy (18) & $\begin{array}{c}2,377 \\
(2,020-2,734) \\
\end{array}$ & $\begin{array}{c}0,844 \\
(0,815-0,872) \\
\end{array}$ & 0,9977 & $\begin{array}{c}2,828 \\
(2,210-3,446) \\
\end{array}$ & $\begin{array}{c}0,855 \\
(0,801-0,909)\end{array}$ & 0,9918 \\
\hline 4 Cuinchy (62) & $\begin{array}{c}2,455 \\
(2,030-2,880)\end{array}$ & $\begin{array}{c}0,863 \\
(0,830-0,896)\end{array}$ & 0,9971 & $\begin{array}{c}2,819 \\
(1,981-3,657)\end{array}$ & $\begin{array}{c}0,927 \\
(0,854-1,000)\end{array}$ & 0,9879 \\
\hline 5 Lizy-sur-Ourcq (77) & $\begin{array}{c}0,814 \\
(0,532-1,097) \\
\end{array}$ & $\begin{array}{c}0,912 \\
(0,849-0,976) \\
\end{array}$ & 0,9902 & $\begin{array}{c}0,887 \\
(0,422-1,352)\end{array}$ & $\begin{array}{c}1,026 \\
(0,895-1,156)\end{array}$ & 0,9683 \\
\hline 6 Meximieux (01) & $\begin{array}{c}0,819 \\
(0,575-1,063) \\
\end{array}$ & $\begin{array}{c}0,919 \\
(0,864-0,973) \\
\end{array}$ & 0,9929 & $\begin{array}{c}0,747 \\
(0,373-1,122) \\
\end{array}$ & $\begin{array}{c}1,101 \\
(0,977-1,225) \\
\end{array}$ & 0,9758 \\
\hline 7 Milly-la-Forêt (91) & $\begin{array}{c}1,077 \\
(0,983-1,172)\end{array}$ & $\begin{array}{c}0,860 \\
(0,844-0,876)\end{array}$ & 0,9993 & $\begin{array}{c}1,046 \\
(0,625-1,468)\end{array}$ & $\begin{array}{c}1,068 \\
(0,962-1,174)\end{array}$ & 0,9819 \\
\hline 8 Versailles INRA (78) & $\begin{array}{c}2,190 \\
(1,900-2,490)\end{array}$ & $\begin{array}{c}0,743 \\
(0,718-0,768)\end{array}$ & 0,9974 & $\begin{array}{c}3,640 \\
(3,166-4,114)\end{array}$ & $\begin{array}{c}0,746 \\
(0,711-0,781)\end{array}$ & 0,9950 \\
\hline
\end{tabular}


TABLEAU 4

Adsorption de l'UKJ-1506 par différents sols: variation des paramètres des isothermes d'adsorption de FREUNDLICH ( $x / m=$ KC $/ / n$ ) en fonction de la gamme des concentrations initiales. La valeur estimée de chaque paramètre est accompagnée de l'intervalle de confiance approché à $95 \mathrm{p} .100$.

Adsorption of UKJ-1506 by different soils : variation of the parameters of the FREUNDLICH adsorption isotherms ( $x / m=K C / \mathrm{m})$ according to the range of initial concentrations. The estimated value for each parameter is given with its $95 \%$ confidence interval.

\begin{tabular}{|c|c|c|c|c|c|c|c|c|}
\hline \multirow{2}{*}{$\begin{array}{l}\text { Gamme des concen- } \\
\text { trations initiales } \\
\text { LS }\end{array}$} & \multirow{2}{*}{$\frac{0,1-1 \mu \mathrm{g} / \mathrm{ml}}{\mathrm{K}}$} & \multicolumn{3}{|c|}{$1-10 \mu \mathrm{g} / \mathrm{ml}$} & \multirow{2}{*}{$\frac{10-320 \mu \mathrm{g} / \mathrm{ml}}{\mathrm{K}}$} & \multicolumn{3}{|c|}{$0,1-320 \mu \mathrm{g} / \mathrm{ml}$} \\
\hline & & $1 / n$ & $\mathrm{~K}$ & $1 / n$ & & $1 / n$ & $\mathrm{~K}$ & $1 / \mathrm{n}$ \\
\hline 1 Laval-en-Brie (77) & $\begin{array}{c}1,088 \\
(1,028-1,149)\end{array}$ & $\begin{array}{c}0,819 \\
(0,748-0,890)\end{array}$ & $\begin{array}{c}1,154 \\
(0,803-1,505)\end{array}$ & $\begin{array}{c}0,784 \\
(0,618-0,949)\end{array}$ & $\begin{array}{c}1,275 \\
(0,484-2,067)\end{array}$ & $\begin{array}{c}0,830 \\
(0,715-0,944)\end{array}$ & $\begin{array}{c}1,246 \\
(0,782-1,710)\end{array}$ & $\begin{array}{c}0,834 \\
(0,766-0,903\end{array}$ \\
\hline 2 Villars-les-Dombes $(01)$ & $\begin{array}{c}1,104 \\
(0,872-1,336)\end{array}$ & $\begin{array}{c}0,853 \\
(0,579-1,126)\end{array}$ & $\begin{array}{c}1,147 \\
(1,047-1,249)\end{array}$ & $\begin{array}{c}0,955 \\
(0,906-1,005)\end{array}$ & $\begin{array}{c}1,153 \\
(0,620-1,687)\end{array}$ & $\begin{array}{c}0,875 \\
(0,790-0,961)\end{array}$ & $\begin{array}{c}1,165 \\
(0,842-1,488)\end{array}$ & $\begin{array}{c}0,873 \\
(0,822-0,924)\end{array}$ \\
\hline 3 Azy (18) & $\begin{array}{c}2,286 \\
(1,954-2,618)\end{array}$ & $\begin{array}{c}0,901 \\
(0,754-1,048)\end{array}$ & $\begin{array}{c}2,175 \\
(1,729-2,622)\end{array}$ & $\begin{array}{c}0,976 \\
(0,843-1,109)\end{array}$ & $\begin{array}{c}2,371 \\
(1,777-2,966)\end{array}$ & $\begin{array}{c}0,844 \\
(0,797-0,892)\end{array}$ & $\begin{array}{c}2,377 \\
(2,020-2,734)\end{array}$ & $\begin{array}{c}0,844 \\
(0,815-0,872)\end{array}$ \\
\hline 4 Cuinchy (62) & $\begin{array}{c}3,008 \\
(2,643-3,373)\end{array}$ & $\begin{array}{c}0,791 \\
(0,693-0,890)\end{array}$ & $\begin{array}{c}3,020 \\
(2,253-3,787)\end{array}$ & $\begin{array}{c}0,987 \\
(0,801-1,172)\end{array}$ & $\begin{array}{c}2,399 \\
(1,721-3,576)\end{array}$ & $\begin{array}{c}0.867 \\
(0,813-0,921)\end{array}$ & $\begin{array}{c}2,455 \\
(2,030-2,880)\end{array}$ & $\begin{array}{c}0,863 \\
(0,830-0,896)\end{array}$ \\
\hline 5 Lizy-sur-Ourcq (77) & $\begin{array}{c}0,935 \\
(0,866-1,004)\end{array}$ & $\begin{array}{c}0,818 \\
(0,714-0,922)\end{array}$ & $\begin{array}{c}1,030 \\
(0,723-1,337)\end{array}$ & $\begin{array}{c}0,950 \\
(0,786-1,113)\end{array}$ & $\begin{array}{c}0,793 \\
(0,338-1,247)\end{array}$ & $\begin{array}{c}0,917 \\
(0,813-1,022)\end{array}$ & $\begin{array}{c}0,814 \\
(0,532-1,097)\end{array}$ & $\begin{array}{c}0,912 \\
(0,849-0,976)\end{array}$ \\
\hline 6 Meximieux (01) & $\begin{array}{c}1,122 \\
(0,981-1,264)\end{array}$ & $\begin{array}{c}0,801 \\
(0,642-0,901)\end{array}$ & $\begin{array}{c}1,072 \\
(0,821-1,324)\end{array}$ & $\begin{array}{c}0,979 \\
(0,849-1,109)\end{array}$ & $\begin{array}{c}0,794 \\
(0,404-1,184)\end{array}$ & $\begin{array}{c}0,925 \\
(0,835-1,014)\end{array}$ & $\begin{array}{c}0,819 \\
(0,575-1,063)\end{array}$ & $\begin{array}{c}0,919 \\
(0,864-0,973)\end{array}$ \\
\hline 7 Milly-la-Forêt (91) & $\begin{array}{c}1,598 \\
(0,969-2,228)\end{array}$ & $\begin{array}{c}1.343 \\
(0,718-1,967)\end{array}$ & $\begin{array}{c}1,260 \\
(0,816-1,704)\end{array}$ & $\begin{array}{c}0,776 \\
(0,583-0,969)\end{array}$ & $\begin{array}{c}1,083 \\
(0,929-1,237)\end{array}$ & $\begin{array}{c}0,859 \\
(0,833-0,885)\end{array}$ & $\begin{array}{c}1,077 \\
(0,983-1,172)\end{array}$ & $\begin{array}{c}0,860 \\
(0,844-0,876)\end{array}$ \\
\hline 8 Versailles-INRA (78) & $\begin{array}{c}1,175 \\
(1,102-1,248)\end{array}$ & $\begin{array}{c}0,823 \\
(0,745-0,901)\end{array}$ & $\begin{array}{c}1,421 \\
(1,068-1,774)\end{array}$ & $\begin{array}{c}0,836 \\
(0,695-0,976)\end{array}$ & $\begin{array}{c}2,312 \\
(1,840-2,783)\end{array}$ & $\begin{array}{c}0,733 \\
(0,695-0,772)\end{array}$ & $\begin{array}{c}2,190 \\
(1,900-2,490)\end{array}$ & $\begin{array}{c}0,743 \\
(0,718-0,768)\end{array}$ \\
\hline
\end{tabular}

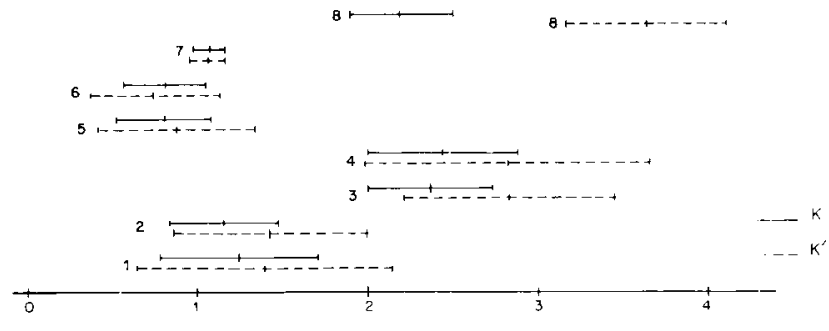

Figure 3

Comparaison des coefficients d'adsorption $K$ et de désorption $K^{\prime}$ de I'UKJ-1506 en fonction du type de sol; les valeurs estimées de $K$ et $K^{\prime}$ sont représentées avec leur intervalle de confiance approché à 95 p. 100.

Comparison of the adsorption and desorption coefficients $K$ and $K^{\prime}$ for UKJ-1506 according to soil type; the estimated values of $K$ and $K^{\prime}$ are represented with their $95 \%$ confidence interval.

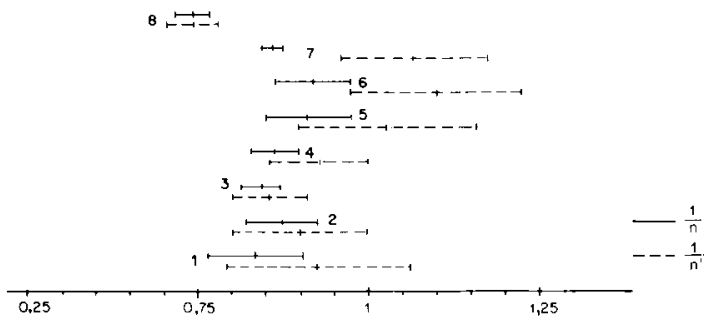

Figure 4

Comparaison des paramètres $1 / n$ et $1 / n^{\prime}$ des isothermes d'adsorption et de désorption de l'UKJ-1506 en fonction du type de sol; les valeurs estimées de $1 / n$ et $1 / n^{\prime}$ sont représentées avec leur intervalle de confiance approché à $95 \mathrm{p} .100$.

Comparison of the parameters $1 / n$ and $1 / n^{\prime}$ of the adsorption and desorption isotherms of UKJ-1506 according to soil type; the estimated values of $1 / n$ and $1 / n^{\prime}$ are represented with their $95 \%$ confidence interval.

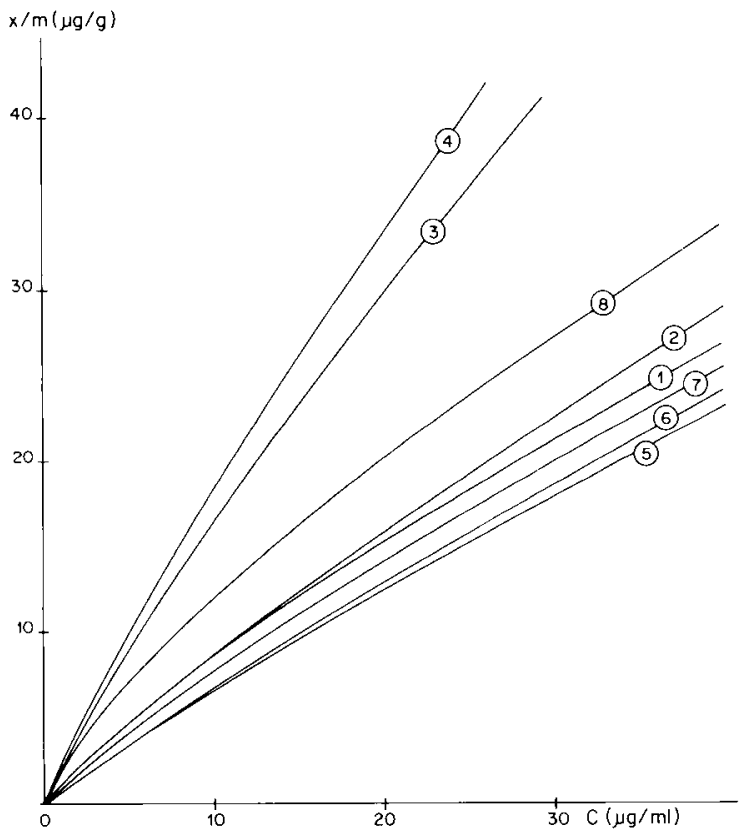

Figure 5

Isothermes d'adsorption de l'UKJ-1506 obtenues par ajustement des données expérimentales au modèle empirique de FREUNDLICH $\left(x / m=K C^{l / n}\right)$.

UKJ-1506 adsorption isotherme obtained by fitting the experimental values to the empirical FREUNDLICH Model $\left(x / m=K C^{1 / n}\right)$.

Sols :

Soils :

$1:$ Laval-en-Brie (77)

$2:$ Villars-les-Dombes (01)

3: Azy (18)

4 : Cuinchy (62)

$5:$ Lizy-sur-Ourcq (77)

6: Meximieux (01)

7: Milly-la-Forêt (91)

$8:$ INRA Versailles (78) 
vent être considérées comme statistiquement identiques (en réalité, les intervalles de confiance des valeurs de $1 / \mathrm{n}$ du sol de Versailles et de Laval-en-Brie se recoupent très légèrement; tabl. 3). Le fait que 7 des 8 valeurs de $1 / \mathrm{n}$ puissent être considérées comme identiques autorise la comparaison des coefficients d'adsorption $\mathrm{K}$ correspondants.

Lorsque nous avons présenté les premiers résultats concernant l'adsorption de l'UKJ-1506, l'estimation des paramètres $K$ et $1 / \mathrm{n}$ avait été réalisée par régression linéaire (JAMET \& WOLFF, 1981) ; l'équation 2 étant linéarisée par une transformation logarithmique (JAMET \& PIEDALLU, 1975a).

Ces premières estimations nous ont servi de valeurs d'entrée lors de l'utilisation du programme de régression non linéaire HAUSS59 (JOLIVET, 1983). Par rapport à ces premières estimations les valeurs du tableau 3 présentent, d'une façon générale, de faibles variations ; cependant la différence la plus prononcée s'observe pour le sol de Versailles. Les premières estimations des paramètres $K$ et $1 / n$ effectuées par régression linéaire (JAMET \& WOLFF, 1981) étaient égales respectivement à 1,28 et 0,87 . Les valeurs estimées par régression non-linéaire (tabl. 3) sont respectivement 2,19 et 0,743 . L'isotherme de FREUNDLICH a une courbure plus prononcée, et la valeur du coefficient d'adsorption $\mathrm{K}$ est nettement plus élevée. La régression non linéaire n'est pas seulement mieux adaptée mais elle est, sur le plan statistique, nécessaire pour l'estimation des paramètres $K$ et $1 / \mathrm{n}$ car le modèle de FREUNDLICH est un modèle non linéaire.

L'examen des valeurs estimées de $\mathrm{K}$ permet de regrouper les sols étudiés de la manière suivante :

- sols $1,2,5,6$ et $7:$ la valeur de $\mathrm{K}$ est proche de l'unité, ce qui traduit une faible adsorption de l'UKJ1506 ; les isothermes correspondantes sont statistiquement identiques (les valeurs de $K$ et de $1 / n$ ne diffèrent pas significativement) et leur tracé est très voisin (fig. 5). La teneur de ces 5 sols en matières organiques varie entre 1 et 2 p. 100 (tabl. 1),

- sols 3 et 4 : le coefficient d'adsorption $K$ est pro- che de 2,5 ce qui indique une adsorption plus importante de l'UKJ-1506 sur ces sols dont la teneur en matières organiques est respectivement de 4 et 5,37 p. 100 ,

- sol 8 : il se distingue des autre sols à la fois par sa valeur de $K$, proche de 2 et celle de $1 / \mathrm{n}(0,743)$ nettement inférieure aux autres. Le tracé de l'isotherme correspondante se situe entre ceux des deux groupes précédents (fig. 5).

Le rôle de la matière organique dans l'adsorption des produits phytosanitaires a été souligné à maintes reprises; généralement les auteurs de telles études s'appuient sur la détermination du coefficient de corrélation linéaire entre $\mathrm{K}$ et la teneur en matières organiques. Certes, cette façon de procéder est critiquable : un taux de matières organiques ne renseigne pas sur le degré d'évolution de celies-ci donc sur leurs propriétés. Pour la plupart des sols cultivés, il est connu qu'il existe une relation étroite entre l'adsorption des produits phytosanitaires et la teneur des sols en matières organiques (CALVET et al., 1980a); la corrélation observée ici va dans ce sens. Nous avons déjà souligné l'existence d'une corrélation positive et hautement significative $(r=0,995)$ entre le coefficient d'adsorption $\mathrm{K}$ de l'UKJ-1506 et le pourcentage de matières organiques des sols (JAMET \& WOLFF, 1981).

A partir des nouvelles estimations de $K$, nous avons à nouveau examiné la relation entre ce paramètre et la teneur des sols en matières organiques. La distribution des points expérimentaux (fig. 6) suggère l'existence d'une relation logarithmique entre $\mathrm{K}$ et le pourcentage de matières organiques ; c'est pourquoi nous avons testé le modèle suivant :

$$
\mathrm{K}=\mathrm{a}+\mathrm{b}(\mathrm{p} .100 \mathrm{MO})
$$

dans lequel a et $b$ sont deux constantes que nous avons déterminées par régression non linéaire :

- d'abord en tenant compte de tous les sols,

- puis en éliminant le point correspondant au sol de Versailles ; ce qui est logique, compte tenu de ce que

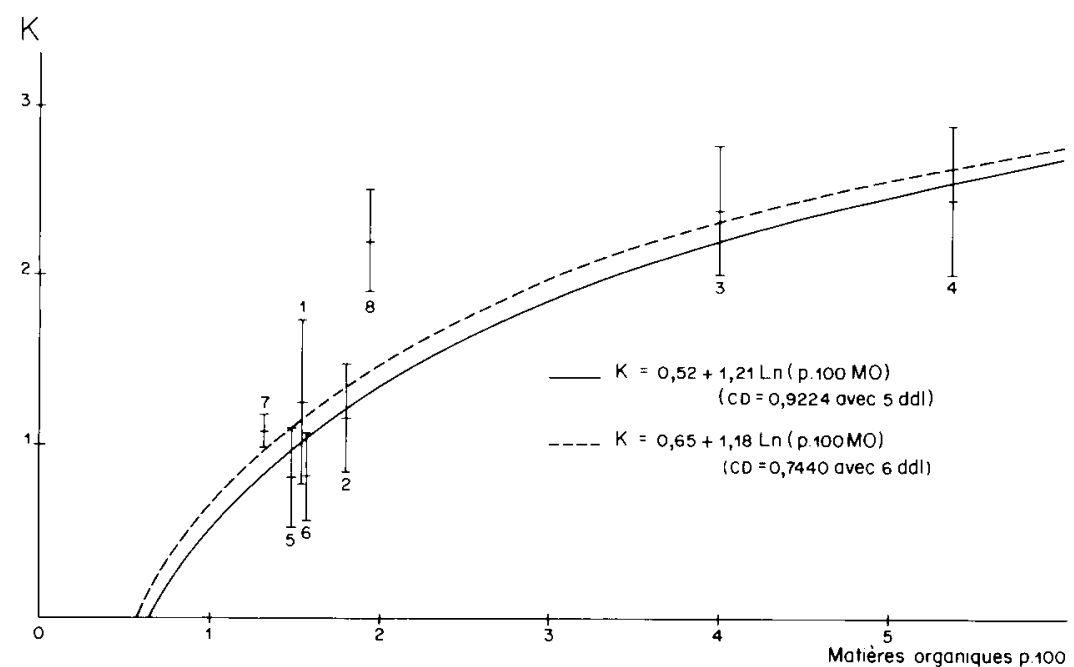

Figure 6

Relation entre le coefficient d'adsorption $K$ et la teneur des sols en matières organiques; les valeurs estimées de $K$ sont représentées avec leur intervalle de confiance approché à $95 \mathrm{p} .100$. Le meilleur ajustement (courbe en trait plein; $C D=0,9224$ ) est obtenu sans tenir compte de la valeur de $K$ du sol 8 (INRA-Versailles).
Relationship between adsorption coefficient $K$ and soil organic matter contents ; the estimated values for $K$ are represented with their $95 \%$ confidence interval. The best fit (solid curve; $D C=0.9224$ ) was obtained without the $K$ value of soil 8 (INRA-Versailles). 
nous avons dit précédemment sur la comparaison des coefficients $\mathrm{K}$ entre eux.

Les résultats de ces modélisations sont regroupés sur la figure 6. On notera :

- la confirmation du rôle de la matière organique dans l'adsorption de l'UKJ-1506; le coefficient de détermination, obtenu avec les sols 1 à 7 est de 0,9224 . Cette valeur traduit également l'intérêt du modèle 3 retenu,

- le regroupement des sols $1,2,5,6$ et 7 , et des sols 3 et 4 , comme cela a été précédemment souligné.

Examinons maintenant le rôle de la gamme de concentrations initiales sur l'estimation des paramètres $K$ et $1 / \mathrm{n}$ (tabl. 4). Pour les sols 1 à 7 , les estimations de $\mathrm{K}$ ne diffèrent pas significativement, quelle que soit la gamme de concentrations initiales utilisée ; les variations observées pour les sols 4 (Cuinchy) et 6 (Meximieux) ne sont pas significatives. Dans le cas du sol 8 (Versailles), on observe une augmentation significative de la valeur estimée de $\mathrm{K}$ lorsqu'on passe de la gamme des faibles concentrations initiales $(0,1-1,0 \mu \mathrm{g} / \mathrm{ml})$ à celles des fortes concentrations initiales (10$320 \mu \mathrm{g} / \mathrm{ml}$ ) ; la valeur estimée avec les fortes concentrations ne diffère pas de celle obtenue avec la totalité des 28 points expérimentaux.

Mais beaucoup plus intéressante nous paraît être l'absence de différence significative entre les estimations de $1 / n$, quelle que soit la gamme de concentrations initiales utilisée ; et cette hypothèse est valable pour tous les sols. Ainsi, les valeurs de $1 / n$ estimées aux faibles et aux fortes concentrations initiales sont statistiquement identiques. Les estimations des $1 / n$ obtenues avec la totalité des points expérimentaux (tabl. 3) indiquent qu'aucune des 8 isothermes ne peut être considérée comme linéaire : la borne supérieure de l'intervalle de confiance approché à 95 p. 100 est toujours inférieure à l'unité. Une telle observation, dans la mesure où elle serait confirmée pour d'autres molécules, devrait retenir l'attention de ceux qui modélisent des courbes d'élution en admettant qu'aux faibles concentrations les isothermes d'adsorption peuvent être considérées comme linéaires.

\section{Les isothermes de désorption}

Pour chaque sol, nous avons déterminé $K^{\prime}$ et $1 / n^{\prime}$ les paramètres des isothermes de désorption ; les estimations obtenues sont regroupées au tableau 3 et comparées à $K$ et $1 / n$ respectivement sur les figures 3 et 4. Mis à part le sol 8 (Versailles), aucun coefficient de désorption $\mathrm{K}^{\prime}$ ne diffère significativement du coefficient d'adsorption $\mathrm{K}$ correspondant (fig. 3) ; ainsi pour les sols 1 à 7, l'adsorption de l'UKJ-1506 est un phénomène réversible, ce qui confirme les résultats obtenus lors de l'étude de l'entraînement par lessivage (JAMET \& WOLFF, 1981). Pour le sol de Versailles, le fait que $K^{\prime}$ soit supérieur à $K$ n'implique pas forcé- ment l'existence d'une hystérèse ; ce résultat peut s'expliquer par une vitesse de désorption très lente. Cette explication paraît d'autant plus satisfaisante que la courbe d'élution correspondante présente une traînée très prononcée (JAMET \& WOLFF, 1981). Dans le cas du sol de Versailles le temps (arbitrairement fixé à $20 \mathrm{~h}$ ) n'est apparemment pas suffisant pour atteindre l'équilibre de désorption. Quant aux valeurs de $1 / n^{\prime}$ elles ne diffèrent significativement de celles de $1 / \mathrm{n}$ que dans les cas des sols 6 (Meximieux) et 7 (Milly-la-Forêt) ; pour ces deux sols le paramètre $1 / n^{\prime}$ est légèrement supérieur à l'unité. Tout comme pour l'adsorption, le modèle de FREUNDLICH (équation 2) permet une description satisfaisante du phénomène de désorption ; les valeurs du coefficient de détermination, même si elles sont toujours inférieures à celles obtenues pour l'isotherme d'adsorption, restent malgré tout très élevées : elles sont toujours supérieures à 0,95 (tabl. 4).

\section{CONCLUSIONS}

Cette étude des caractéristiques de l'adsorption et de la désorption de l'UKJ-1506 précise certains aspects du comportement de ce produit dans le sol; elle souligne également l'intérêt de certaines méthodes :

- Ce nouvel herbicide est rapidement adsorbé dans le sol ; l'équilibre d'adsorption est dans tous les cas atteint au bout de $4 \mathrm{~h}$.

- La quantité adsorbée est d'autant plus importante que la teneur du sol en matières organiques est élevée ; la seule connaissance de cette caractéristique du sol permet une estimation satisfaisante du coefficient d'adsorption $\mathrm{K}$.

- Les isothermes d'adsorption ne sont pas linéaires ; toutes les valeurs de $1 / \mathrm{n}$ diffèrent statistiquement de 1 .

- L'adsorption de l'UKJ-1506 est réversible, et ceci pour tous les sols utilisés dans cette étude.

- L'intérêt de la régression non linéaire, utilisée en particulier pour l'estimation des paramètres $K$ et $1 / n$ du modèle de FREUNDLICH, n'est certes plus à démontrer ; il existe actuellement plusieurs programmes de régression non linéaire: ces programmes, plus ou moins performants, en particulier selon l'algorithme d'optimisation numérique utilisé, sont de toute manière statistiquement nécessaires pour l'estimation des paramètres de modèles non linéaires. C'est pourquoi nous pensons que cette méthode devrait être systématiquement utilisée pour le calcul des isothermes d'adsorption. 


\section{RÉFÉRENCES BIBLIOGRAPHIQUES}

Biggar J. W., Mingelgrin U., Cheung M. W., 1978. Equilibrium and kinetics of adsorption of picloram and parathion with soils. J. Agric. Food Chem., 26, 1306-1312.

Boutemy G., 1980. New thiopyrimidines as herbicides. Proceedings 1980 British Crop Protection Conference, Weeds, p. 47-52.

Calvet R., Graffin Ph., 1974. Mise au point bibliographique : description mathématique des phénomènes de transport des solutions aqueuses dans les milieux poreux. Ann. Agron., 25 (2-3), 123-155.

Calvet R., Jamet P., 1979. Données générales sur le transport des pesticides dans les sols. Trav. Soc. Pharm. Montpellier, 39 (4), 275288.

Calvet R., Tercé Martine. Arvieu J. C.. 1980a. Mise au point bibliographique: Adsorption des pesticides par les sols et leurs constituants. III. Caractéristiques générales de l'adsorption des pesticides. Ann. Agron., 31 (3), 239-257.

Calvet R., Terce Martine, Arvieu J. C., 1980b. Mise au point bibliographique : Adsorption des pesticides par les sols et leurs constituants. V. Les méthodes d'étude de l'adsorption. Ann. Agron., 31 (4), 413-427.

Green R. E., Yamane V. K. (1971). Precision in pesticide adsorption measurements. Soil Sci. Soc. Am. Proc., 34, 353-355.
Jamet P., Piedallu Marie-Andrée, 1975a. Etude de l'adsorption et de la désorption de la pyrazone par différents types de sols. Weed. Res., 15, 113-121.

Jamet P., Piedallu Marie-Andrée, 1975b. Mouvement du carbofuran dans différents types de sol : étude de l'adsorption et de la désorption du carbofuran. Phytiat.-Phytopharm., 24, 279-296.

Jamet P., Wolff Nelly, 1981. Adsorption, désorption et entraînement par lessivage de l'UKJ-1506 (thiopyrimidine herbicide) dans différents sols : premiers résultats. In Proc. EWRS Symp. Theory and Practice of the use of soil applied herbicides, p. 25-53.

Jamet P., Thoisy Jeanne-Chantal, Laredo C., Decoux G., 1984. Etude et modélisation de la cinétique d'adsorption des pesticides dans le sol. Rapport interne (10 août 1984).

Jolivet E., 1983. Introduction aux modèles mathématiques en biologie. Actual. scientif. agronom. l'I.N.R.A. ${ }^{\circ} 11$, Masson éd., Paris, $151 \mathrm{p}$.

Kerhoas L., Dubroca Jacqueline, 1984. Etude de la dégradation d'un herbicide dans le sol : essais de laboratoire et essais de plein champ. In Actes symposium intern. "Ecotoxicologie terrestre" (12-14/12/84), p. 51-67, Les Arcs (Savoie) France. Ministère de l'Environnement Paris. 